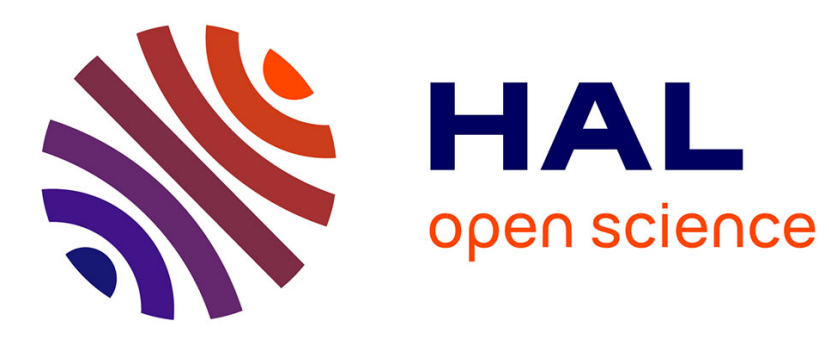

\title{
Investigations for a model reduction technique of fluid-structure coupled systems
}

Brahim Ben Smida, R Majed, Noureddine Bouhaddi, Morvan Ouisse

\section{To cite this version:}

Brahim Ben Smida, R Majed, Noureddine Bouhaddi, Morvan Ouisse. Investigations for a model reduction technique of fluid-structure coupled systems. Proceedings of the Institution of Mechanical Engineers, Part C: Journal of Mechanical Engineering Science, 2012, 226 (1), pp.42-54. 10.1177/0954406211411863. hal-02300121

\section{HAL Id: hal-02300121 \\ https://hal.science/hal-02300121}

Submitted on 4 Apr 2020

HAL is a multi-disciplinary open access archive for the deposit and dissemination of scientific research documents, whether they are published or not. The documents may come from teaching and research institutions in France or abroad, or from public or private research centers.
L'archive ouverte pluridisciplinaire HAL, est destinée au dépôt et à la diffusion de documents scientifiques de niveau recherche, publiés ou non, émanant des établissements d'enseignement et de recherche français ou étrangers, des laboratoires publics ou privés. 


\title{
Investigations for a model reduction technique of fluid-structure coupled systems
}

\author{
B Ben Smida ${ }^{1 *}$, R Majed $^{1}, \mathbf{N}_{\text {Bouhaddi }}{ }^{2}$, and M Ouisse ${ }^{2}$ \\ ${ }^{1}$ Preparatory Engineering Institute of Nabeul (IPEIN), M'rezgua, Nabeul, Tunisia \\ ${ }^{2}$ FEMTO-ST Institute, Applied Mechanics Laboratory, UMR CNRS 6174, 24, Besançon, France
}

\begin{abstract}
In this paper, a methodology for coupled fluid-structure model reduction is proposed. The overall objective of the method is to reduce the numerical computational costs without affecting the accuracy level of the prediction. This methodology is organized according to the three following steps. In the first step, this method uses a reliable criterion for selecting the number of the kept modes for the fluid and the structure in vacuo subsystems. In the second step, this basis will be enriched through static residual responses taking into account the fluidstructure coupling effects. These responses are selected according to an energetic criterion. Finally, the enriched basis is extended by introducing some residual static responses due to the error forces considered as structural modifications forces.

In the context of the finite element method (FEM), the performances of the proposed method are established through a comparative study with other strategies that are proposed in the literature. Thus, the computational cost (CPU time) and the accuracies of the different methods are discussed and compared with a reference method. The validation of the proposed method and the comparative study are performed through two numerical simulation examples. The first one concerns a parallelepiped acoustic cavity with a simply supported plate. The method can handle both weak and strong couplings; as illustrated in the examples. The second one consists of a pipe with a strong coupling and a larger model size.
\end{abstract}

Keywords: vibroacoustic, cavity, reduction method, cost, fluid-structure coupling

\section{INTRODUCTION}

Nowadays, new commercial and normative strategies are based on the customer orientation principle. In the automotive and aerospace sectors, acoustic and vibration comfort is one of the most important benefits for customer satisfaction. In this context, the mastering of design parameters becomes an essential key point in the creation process of new products. The fluidstructure interaction in various mechanical systems leads to mathematical models that are analytically

\footnotetext{
*Corresponding authors: B. Ben Smida, Preparatory Engineering Institute of Nabeul (IPEIN), 8000 M'rezgua, Nabeul, Tunisia. email: ibensmi@gmail.com
}

difficult to solve. Only few situations lead to analytical results, among which an acoustic cavity coupled to a plate, such as that discussed by Pretlove [1]. Most of the time, for industrial applications, numerical simulation tools are mandatory for predicting behaviour and to verify compliance with design objectives. In this paper we will focus on internal problems, namely an acoustic cavity closed by an elastic structure. In this context, the finite element method (FEM) is the most popular technique, even if boundary elements can also be used, as mentioned by Fischer [2] .

One can distinguish several formulations in the elaboration of the equations governing the fluidstructure interaction problem. These formulations are characterised by the state variable of the 
corresponding fluid domain $[\mathbf{3}, \mathbf{4}, \mathbf{5}]$. The formulation using the pressure field $\mathrm{p}$, which is simple to implement and commonly used in commercial computer codes, is not well posed for the static case. A constraint relating the pressure and the displacement of the wall is taken into account [6] to overcome the indeterminacy problem.

The major drawback of this formulation is the asymmetry of the global coupled matrices. A symmetric form of the model can be derived by a simple transformation proposed by Irons [7]. This transformation is penalized by the loss of the sparse matrices character, which implies storage and computation time problems. Moreover, when the considered problem requires filtering of rigid body modes, this transformation necessitates a specific filtering procedure. The symmetrization of the coupled system matrices is also discussed by Felippa [8]. In all cases, the global calculation cost remains high, so many research works are dedicated to the development of model reduction techniques in order to reduce the computational cost without affecting the numerical accuracy $[9,10,11,12,13,14]$.

Boily et al. [14] demonstrated that modal truncation is very important in the prediction of the pressure field. The truncation criterion $n \times f_{\max }$, generally used in structural dynamics with $n=2$ or 3 , is not sufficient for selecting the structure and fluid subbases. The authors proposed a criterion for selecting the modes based on the exploitation of the coupling terms. Tournour et al. [15] have shown that the use of a decoupled modal basis leads to a poor convergence in the case of high rank modes of a subsystem that can be coupled with those of another subsystem. This problem is due to the lack of a reliable criterion for the choice of the selected modes for each subsystem. The convergence of the method is improved by using a pseudostatic correction for the structure and the fluid.

Bobillot [11] has proposed an iterative technique to improve the initial truncated basis by using residual static vectors. A stop criterion is used when the precision of enrichment reaches a chosen tolerance. Maess [16] shows that, for the resolution of the eigenvectors problem, the matrices are badly conditioned and proposes to use a preconditioning technique. The sub-bases are enriched using the same methodology as that proposed by Bobillot [11]. Tran et al. [9] proposed to operate through decoupled sub-bases that are enriched by the responses of static residual forces. This technique is extended to sub-structuring in order to deal with large vibroacoustics-damped problems.

These studies show that the use of uncoupled modal bases leads to a bad convergence rate. The $n \times f_{\max }$ criterion, which is generally used in structural dynamics, has two main disadvantages. On one hand, there is a lack of information on the basis used, since it does not take into account the effects of the higher rank coupled modes. On the other hand, the unnecessary retained modes increase the computing time without any valuable information. At this stage, several questions require answers: What are the modes that should be kept in the bases? How should the missing information be completed? In order to answer these questions, measurable and specific criteria are required.

In this context, the method proposed in this paper is an alternative technique to overcome several difficulties encountered in the reduction of the fluidstructure coupled model. In this method, the use of a set of consistent criteria and corrections is proposed. At first, the initial uncoupled modes are selected. Then, the uncoupled bases are extended by using coupled static responses according to an energy criterion. Finally, a stop criterion based on the static residual response is used. The presented methodology is illustrated through two numerical examples. The first one illustrates the performance of the proposed method compared with other methods from the literature for both weak and strong coupling. The second example shows the advantages of the method for a more complex structure with a relatively large finite element model.

\section{VIBROACOUSTIC MODEL}

In this study, the fluid-structure problem is based on classical equations corresponding to a linear description of vibroacoustics phenomena [4]. For a formulation based on displacement-pressure formulation $(u, p)$, the behaviour of the fluid-structure undamped coupled system is described by the following system of equations:

$$
\left[\begin{array}{cc}
M_{s} & 0 \\
\rho_{f} C^{T} & M_{f}
\end{array}\right]\left\{\begin{array}{l}
\ddot{u} \\
\ddot{p}
\end{array}\right\}+\left[\begin{array}{cc}
K_{s} & -C \\
0 & K_{f}
\end{array}\right]\left\{\begin{array}{l}
u \\
p
\end{array}\right\}=\left\{\begin{array}{c}
F_{s} \\
0
\end{array}\right\}
$$

where $K_{s}, M_{s}$ are respectively the stiffness matrix and the mass matrix of the structure, $K_{f}$ and $M_{f}$ are respectively the matrices corresponding to the discretization of the kinetic energy and compressibility of the fluid. $C$ is the coupling matrix. $F_{s}$ is the external load applied to the structure.

\section{RESOLUTION METHODS}

The solution of the equations (1) can be performed by a variety of methods presented in this section. The aim of the present study is to find a reduction basis 
to minimize the computational cost with sufficient precision in the prediction. The modal basis of the structure and the cavity classically uncoupled and truncated must be enriched to take into account the coupling effects and the non-calculated modes of higher rank. Generally, the structure modal basis is truncated following the frequency criterion $f_{s}=n \times f_{\max } \quad\left(f_{\max }\right.$ is the maximum frequency of interest) while this criterion is not sufficient for the cavity modal basis. Therefore, some modes that are important, coupled with those of the structure, may be eliminated.

\subsection{Direct method}

The transformation of equation (1) leads to the following form of the coupled system model:

$$
\left(-\omega^{2}\left[\begin{array}{cc}
M_{s} & 0 \\
\rho_{f} C^{T} & M_{f}
\end{array}\right]+\left[\begin{array}{cc}
K_{s} & -C \\
0 & K_{f}
\end{array}\right]\right)\left\{\begin{array}{l}
U \\
P
\end{array}\right\}=\left\{\begin{array}{c}
F_{s} \\
0
\end{array}\right\}
$$

The direct inversion of the dynamic stiffness allows the computation of the following response (3):

$$
\left\{\begin{array}{l}
U \\
P
\end{array}\right\}=\left(-\omega^{2}\left[\begin{array}{cc}
M_{s} & 0 \\
\rho_{f} C^{T} & M_{f}
\end{array}\right]+\left[\begin{array}{cc}
K_{s} & -C \\
0 & K_{f}
\end{array}\right]\right)^{-1}\left\{\begin{array}{c}
F_{s} \\
0
\end{array}\right\}
$$

The inversion performed at each frequency step can be extremely time consuming and impractical for large models. However, for comparative study, this method will be considered as the reference.

\subsection{Uncoupled bases}

The resolution of the eigenvalue problem for the structure and fluid uncoupled systems allows the computation of the uncoupled modal bases:

$$
\left\{\begin{array}{l}
\left(-\omega^{2} M_{s}+K_{s}\right) U=0 \\
\left(-\omega^{2} M_{f}+K_{f}\right) P=0
\end{array} \Rightarrow\left[T_{o}\right]=\left[\begin{array}{cc}
T_{s o} & 0 \\
0 & T_{f o}
\end{array}\right]\right.
$$

The projection of equation (2) on the truncated modal bases leads to a reduced model as follows:

$$
\left(-\omega^{2}\left[\begin{array}{cc}
\tilde{M}_{s} & 0 \\
\rho_{f} \tilde{C}^{T} & \tilde{M}_{f}
\end{array}\right]+\left[\begin{array}{cc}
\tilde{K}_{s} & -\tilde{C} \\
0 & \tilde{K}_{f}
\end{array}\right]\right)\left\{\begin{array}{c}
q_{s} \\
q_{f}
\end{array}\right\}=\left\{\begin{array}{c}
\tilde{F}_{s} \\
0
\end{array}\right\}
$$

where $\tilde{K}_{s}=T_{s 0}^{T} K_{s} T_{s 0}, \quad \tilde{M}_{s}=T_{s 0}^{T} M_{s} T_{s 0}, \quad \tilde{K}_{f}=T_{f 0}^{T} K_{f} T_{f 0}$, $\tilde{M}_{f}=T_{f 0}^{T} M_{f} T_{f 0}, \quad \tilde{C}=T_{s 0}^{T} C T_{f 0}, \quad \tilde{F}_{s}=T_{s 0}^{T} F_{s}, \quad U=T_{s 0} q_{s}$ and $P=T_{f 0} q_{f}$.

This superposition method is classically used in structural dynamics. It reduces considerably the computation time. Its main disadvantage for a coupled system is the ignorance of the effect of coupled modes, which leads to a very low convergence.

\subsection{Uncoupled bases with modes selection}

This method proposed by Boily et al. $[\mathbf{1 3}, \mathbf{1 4}]$ consists on a priori selection of modes that contribute significantly to the coupled response. A selection criterion is evaluated for each mode. This criterion takes into account the coupling terms, the dynamic participation factors of the modes, and the frequencies separation factor. To consider the dissipative case, a structural damping ratio is introduced in the model denoted as $\eta_{s}$ for the structure and $\eta_{f}$ for the fluid. The structure and fluid matrices are respectively written as

$$
\bar{K}_{s}=\left(1+j \eta_{s}\right) \tilde{K}_{s}, \quad \bar{K}_{f}=\left(1+j \eta_{f}\right) \tilde{K}_{f}
$$

The use of equations (6) and (4) leads to

$$
\left(-\omega^{2}\left[\begin{array}{cc}
\tilde{M}_{s} & 0 \\
\rho_{f} \tilde{C}^{T} & \tilde{M}_{f}
\end{array}\right]+\left[\begin{array}{cc}
\bar{K}_{s} & -\tilde{C} \\
0 & \bar{K}_{f}
\end{array}\right]\right)\left\{\begin{array}{c}
q_{s} \\
q_{f}
\end{array}\right\}=\left\{\begin{array}{c}
\tilde{F}_{s} \\
0
\end{array}\right\}
$$

Equation (7) yields to the calculation of the fluid generalized vector $q_{f_{j}}$

$$
\left\{q_{f_{j}}\right\}=\frac{-\omega^{2} \tilde{C}_{i j}}{\omega^{2}-\omega_{f j}^{2}\left(1+j \eta_{f}\right)} \cdot\left(\frac{T_{0 s i}^{T} F_{s}}{\omega^{2}-\omega_{s i}^{2}\left(1+j \eta_{s}\right)}\right)
$$

This equation shows that a large coupling effect between acoustic mode $j$ and structural mode $i$ occurs if the following conditions are met: the coupling term $\tilde{C}_{i j}$ is large, the structural mode $i$ is excited and the eigenfrequency of both modes are close one to another. This indicator will be used as an a priori selection criterion of the modes retained in the initial truncated bases.

\subsection{Uncoupled bases extended by residual modes iteration}

This method is proposed by Bobillot [11], where the displacement field $U$ and the pressure field $P$ are searched in different subspaces. For each iteration $k, U$, and $P$ are recalculated in the subspaces $T_{s}^{k}$ and $T_{f}^{k}$. A force residual is defined for the displacement field in the reduced form

$$
\left\{R_{L, s, j}^{k}\right\}=\tilde{K}_{s} \tilde{U}_{j}-\tilde{C} \tilde{P}_{j}-\omega_{j}^{2} \tilde{M}_{s} \tilde{U}_{j}
$$

where, $\tilde{U}_{j}=T_{s}^{k} q_{s}$ et $\tilde{P}_{j}=T_{f}^{k} q_{f}$.

Similarly, one can define a force residuals for the pressure field in the form

$$
\left\{R_{L, p, j}^{k}\right\}=\tilde{K}_{f} \tilde{P}_{j}-\omega_{j}^{2} \tilde{C} \tilde{U}_{j}-\omega_{j}^{2} \tilde{M}_{f} \tilde{P}_{j}
$$

The reduced matrices $\tilde{K}_{s}, \tilde{M}_{s}, \tilde{K}_{f}, \tilde{M}_{f}, \tilde{C}$, are defined by equations (5).

These force residuals lead to the computation of the displacement residuals, as follows: 


$$
\begin{array}{ll}
\left\{R_{D, s, j}^{k}\right\}=\left[\hat{K}_{s}\right]^{-1} R_{L, s, j}^{k} \quad \text { where } & \hat{K}_{s}=\tilde{K}_{s}+\alpha_{s} \tilde{M}_{s} \\
\left\{R_{D, f, j}^{k}\right\}=\left[\hat{K}_{f}\right]^{-1} R_{L, f, j}^{k} & \hat{K}_{f}=\tilde{K}_{f}+\alpha_{f} \tilde{M}_{f}
\end{array}
$$

$\alpha_{s}, \alpha_{f}$ are the shift coefficients in mass chosen in practical such that $\omega_{\text {flex }}^{2} / 10<\alpha<\omega_{\text {flex }}^{2}$, where $\omega_{\text {flex }}$ is the eigenfrequency of the first flexible mode.

A convergence criterion and error estimate proportional to the strain energy is used for each subdomain:

$$
\begin{gathered}
\varepsilon_{s, j}^{k}=\frac{\left\|\left\{R_{D, s, j}^{k}\right\}\right\|^{2}}{\left\|T_{s}^{k} q_{s}\right\|^{2}} \geq \operatorname{Tol} \Rightarrow T_{s}^{k+1}=\left[T_{s}^{k},\left\{R_{D, s, j}^{k}\right\}\right] \\
\varepsilon_{f, j}^{k}=\frac{\left\|\left\{R_{D, f, j}^{k}\right\}\right\|^{2}}{\left\|T_{f}^{k} q_{f}\right\|^{2}} \geq \mathrm{Tol} \Rightarrow T_{f}^{k+1}=\left[T_{f}^{k},\left\{R_{D, f, j}^{k}\right\}\right]
\end{gathered}
$$

\subsection{Extended Ritz basis}

The method proposed by Tran et al [9] is based on the use of uncoupled modal bases enriched by residual responses. The second line of equation (2) is rewritten as

$$
\left(K_{f}-\omega^{2} M_{f}\right) P=\omega^{2} \rho_{f} C^{T} U=F_{f s}
$$

The external force corresponding to the coupling effects is written as

$$
F_{f s}=\omega^{2} \rho_{f} C^{T} U
$$

The static response is used for the determination of residual vectors. Since the matrix $K_{f}$ is singular, a shifted stiffness matrix is used in the procedure to eliminate the singularity by adding $\omega_{c}^{2} M_{f}$ with $\omega_{c} \in\left[\omega_{\min }-\omega_{\max }\right]$. The external force $F_{f s}$ caused by the structure in the equation (15) is estimated by introducing the approximate field $T_{s_{0}} q_{s}$ in equation (14). The residual vectors will be written in the following form:

$$
P=\left(K_{f}-\omega_{c}^{2} M_{f}\right)^{-1} \omega_{c}^{2} \rho_{f} C^{T} T_{s 0} q_{s}
$$

For a chosen $\omega_{c}, \omega_{c}^{2} \rho_{f}$ will be constant and can be introduced in the generalized coordinate vector $q_{s}$. Thus, the residual vectors can be written in the following form:

$$
\Delta T_{f s}=\left(K_{f}-\omega_{c}^{2} M_{f}\right)^{-1} C^{T} T_{s 0}
$$

The enriched fluid basis is written as

$$
T_{f}=\left[\begin{array}{ll}
T_{f 0} & \Delta T_{f s}
\end{array}\right]
$$

Generally, the vectors are normalized and orthogonalized. A singular value decomposition is used to ensure a good conditioning of the problem. In the case of strong coupling, the same procedure can also be performed to enrich the structural basis.

\subsection{Proposed reduction method}

The choice of the initial basis is very important because it must contain the maximum information for the coupled system. Each decision step of the method is justified by a specific and measurable selection criterion. This method is based on the exploitation of a truncated uncoupled modal basis for the choice of the structure and fluid modes $n_{s}^{0}$ et $n_{f}^{0}$. The modes kept in the basis are not all useful. Therefore, a priori selection of modes is done by the selection criterion presented in equation (8). Among the selected modes, the truncation is carried out under the following energy criteria:

$$
\begin{aligned}
E_{s, i} & =\frac{T_{s 0}^{T}(i) K_{s} T_{s 0}(i)}{\sum_{i=1}^{n_{s}^{0}}\left(T_{s, s e l}^{T}(i) K_{s} T_{s, s e l}(i)\right)} \geq T o l \\
& \Rightarrow T_{s, i+1}^{s e l}=\left[\begin{array}{ll}
T_{s, i}^{s e l} & T_{s 0}(i)
\end{array}\right] \\
E_{f, i} & =\frac{T_{f 0}^{T}(i) K_{f} T_{f 0}(i)}{\sum_{i=1}^{n_{s}^{0}}\left(T_{f, s e l}^{T}(i) K_{f} T_{f, s e l}(i)\right)} \geq T o l \\
& \Rightarrow T_{f, i+1}^{s e l}=\left[\begin{array}{ll}
T_{f, i}^{s e l} & T_{f 0}(i)
\end{array}\right]
\end{aligned}
$$

where the index $i$ represents the current selected mode number.

In the next step, the basis is enriched by some vectors that reflect the coupling effect. The development of equation (5) leads to the following equations:

$$
\left\{\begin{array}{l}
\left(-\omega^{2} \tilde{M}_{s}+\bar{K}_{s}\right) q_{s}=F_{s}+C q_{f} \\
\left(-\omega^{2} \tilde{M}_{f}+\bar{K}_{f}\right) q_{f}=\omega^{2} \rho_{f} C^{T} q_{s}
\end{array}\right.
$$

By exploiting the structural eigenvectors, in the second equality of equation (21) one can find the vectors $q_{f, i}$ reflecting the load effects applied by the elastic structure on the fluid domain. In the same way, the use of the fluid eigenvectors in the first equality of equation (21) yields the vector $q_{s, i}$ reflecting the load effects applied by the fluid domain on the structure.

$$
\begin{aligned}
& q_{f, i}=\left(-\omega_{c}^{2} M_{f}+K_{f}\right)^{-1} \omega_{c}^{2} \rho_{f} C^{T} T_{s, i}^{s e l} \\
& q_{s, i}=\left(-\omega_{c}^{2} M_{s}+K_{s}\right)^{-1}\left(F_{s}+C T_{f, i}^{s e l}\right)
\end{aligned}
$$


where $\omega_{c}$ is an eigenfrequency chosen in the frequency band of interest $\left(\omega_{c} \in\left[\omega_{\min }-\omega_{\max }\right]\right)$, and $q_{f, i}, q_{s, i}$ are the vectors used for the enrichment of fluid and structure bases.

The same criteria $E_{s, i}$ and $E_{f, i}$ with tolerance Tol are used for the decision making related to the enrichment and construction of the new enriched basis $T_{E}$ :

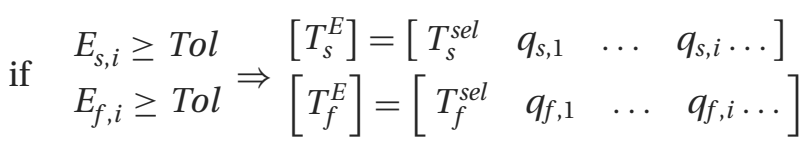

A singular value decomposition (SVD) is useful to ensure the linear independence of the added modes with the initial basis modes.

The enriched basis of the coupled model can finally be written as follow:

$$
T_{E}=\left[\begin{array}{ccc}
T_{s}^{s e l} & \Delta T_{s} & 0 \\
0 & T_{f}^{\text {sel }} & \Delta T_{f}
\end{array}\right]=\left[\begin{array}{cc}
T_{s}^{E} & 0 \\
0 & T_{f}^{E}
\end{array}\right]
$$

If necessary, a final step is considered, in order to fulfil the basis with the missing information corresponding to the static response displacement due to the forces of errors in both structure and fluid domains [11]. In this step, the displacement and pressure responses are estimated by a modal projection

$$
\left\{\begin{array}{l}
\hat{U} \\
\hat{P}
\end{array}\right\}=T_{E}\left\{\begin{array}{l}
q_{s} \\
q_{f}
\end{array}\right\}
$$

The equations (24) and the error loads, called force residuals, obtained by equation (2), lead to the following equations:

$$
\begin{aligned}
& R_{F s}=\left(K_{s}-\omega_{c}^{2} M_{s}\right) \hat{U}-C \hat{P}-F_{s} \\
& R_{F f}=\left(K_{f}-\omega_{c}^{2} M_{f}\right) \hat{P}-\rho_{f} \omega_{c}^{2} C^{T} \hat{U}
\end{aligned}
$$

Unlike the reduction method by projection on an extended iterative residue [11], which assesses the load residuals for each value of $\omega$, the residue computation by equation (26) is performed only once for a value of $\omega_{c}$.

These force residuals are then used to estimate the displacement residuals according to the following form:

$$
\begin{aligned}
& R_{D s}=K_{s}^{-1} R_{F s} \\
& R_{D f}=K_{f}^{-1} R_{F f}
\end{aligned}
$$

The cavity fluid has a rigid body mode which implies that the matrix $K_{f}$ is singular. A classical procedure mode filtering is used to compute the displacement residuals $R_{D f}[\mathbf{1 7}]$.

The enriched sub-bases $T_{s}^{E}$ and $T_{f}^{E}$ will be eventually improved by the displacement residuals vectors

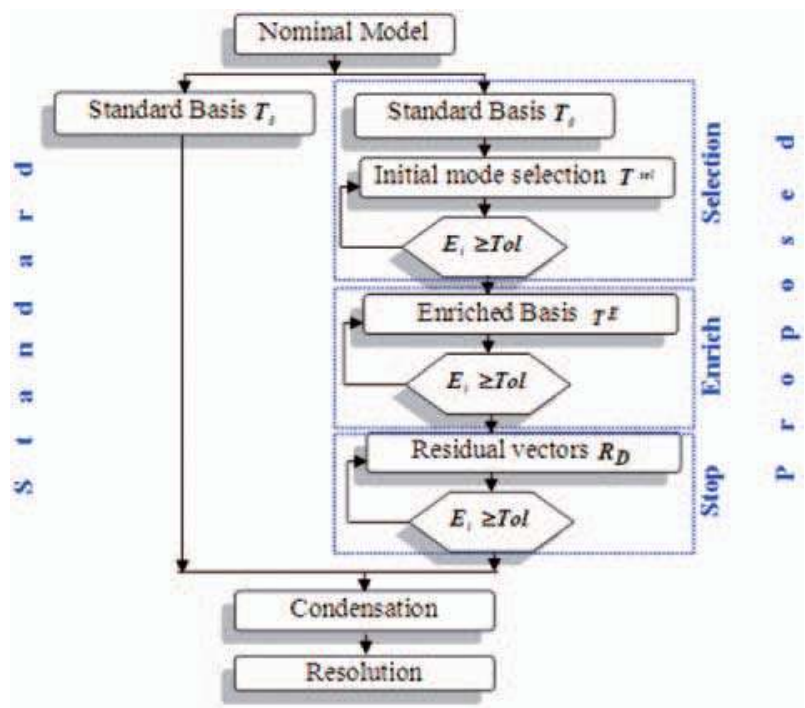

Fig. 1 Main steps of the proposed method

$R_{D s}$ and $R_{D f}$. An SVD is thus necessary. The same indicators estimated at a tolerance $\mathrm{Tol}$, are used as convergence criteria and to check the level of accuracy. This criterion is fundamental in the absence of reference computation by direct approach (case of industrial model).

The main steps of the proposed method are illustrated by the flowchart in Fig. 1.

\section{NUMERICAL VALIDATION STRATEGY}

The performance of the proposed method is evaluated through an investigation in different methods from the literature. The computation time (CPU) and the accuracy of vibroacoustic indicators calculated relatively to the reference method (direct method (DI)) are used. The second method is the projection on an uncoupled basis (DB). The third method is based on the projection on an uncoupled basis with a priori selection of modes (BDS). The fourth method uses enrichment by iteration on the residue (IR). The proposed method in this paper is based on the projection on a uncoupled basis with a priori selection of modes. The last basis is enriched by a set of modes taking into account the coupling effect (BDSE).

The uncoupled basis method (DB) and the uncoupled basis method with a priori selected modes (BDS) do not use truncation criterion. Enrichment by the IR method has its own stop criterion shown in equations $(12,13)$ related to a priori defined tolerance. In order to compare the efficiency of the methods, the same size must be used for all sub-bases. So, the maximum number of vectors in the structure sub-basis ( $\mathrm{Ns}=50$ vectors) and the fluid sub-basis ( $\mathrm{Nf}=150$ vectors) is used as a unified common size limit for all the methods. 


\section{NUMERICAL SIMULATION}

The efficiency of the proposed method is illustrated through two numerical simulation examples. The first example consists in a classical acoustic cavity studied with weak (air) and strong (water) coupling cases. The second example consists in a simplified automotive exhaust system. An Intel(R) Core(TM) 2 Duo CPU T5750@ $2.00 \mathrm{GHz}$ with 2.99 Go RAM is used.

\subsection{Acoustic cavity}

The proposed example is a cube-shaped acoustic cavity filled with air (or water). The upper wall is comprised of a simply supported flexible thin plate. The other walls are rigid. A harmonic point force is located in the coordinates $(x, y, z)=(0.25,0.16,0.00)$ (Fig. 2a). The finite element model consists in $17 \times 14$ structural quadrilateral elements (ANSYS SHELL63 - thin plate) and $17 \times 14 \times 7$ elements acoustic bricks (ANSYS FLUID30). The finite element model includes 3324 degrees of freedom (DOFs) (2160 DOFs of structure, 1164 DOFs of fluid) (Fig. 2b). The mesh size of the finite elements model is based on a criterion of five to six linear elements per mode shape wavelength. This criterion is used by Boily et al. [13].

The structural damping factors introduced respectively for the fluid and structure subsystems are chosen as $\eta_{s}=0.02$ and $\eta_{f}=0.002$. The geometric and physical characteristics are: Plate: $L=0.35 \mathrm{~m}$; $l=0.29 \mathrm{~m} ; E_{0}=0.72 \mathrm{e} 11 \mathrm{~N} / \mathrm{m}^{2} ; r_{\mathrm{s}}=2700 \mathrm{~kg} / \mathrm{m}^{3} ; v=$ 0.3. Cavity: $L=0.35 \mathrm{~m} ; l=0.29 \mathrm{~m} ; h=0.14 \mathrm{~m}$; Air: $r_{\mathrm{f}}=1.2 \mathrm{~kg} / \mathrm{m}^{3} ; c=340 \mathrm{~m} / \mathrm{s}$. Water: $r_{f}=1000 \mathrm{~kg} / \mathrm{m}^{3}$; $c=1500 \mathrm{~m} / \mathrm{s}$.

\subsubsection{Case of cavity filled with air}

For the weak coupling case, the cavity is filled with air. The vibroacoustic analysis is performed in the frequency range $=[0-600 \mathrm{~Hz}]$ containing the first seven modes of the coupled system. This frequency band contains the first four structural modes and the first three acoustic modes of the uncoupled systems (Table 1). It is emphasized that the third cavity mode and the fourth structure mode are coupled around the frequency value of $585 \mathrm{~Hz}$. Figure 3 displays the coupled and uncoupled fluid-structure sub-system eigenfrequencies in the band of interest. It is shown that the third cavity mode and the fourth structure mode are coupled.

Validation of the proposed method. One can start with the construction of uncoupled sub-bases by using equations (4). Two hundred modes are calculated for the structure sub-domain and the same number for the fluid sub-domain ( $n_{s}^{0}=n_{f}^{0}=200$ modes). The choice of the retained modes is made according to the indicator given by equation (8). The use of criteria $E_{s}$ and $E_{f}$ in equations (19) and (20) estimated for a tolerance $\mathrm{Tol}=10^{-3}$ allows the selection of 20 structural modes 90 and fluid modes. The equations (23) associated with the criteria $E_{s}$ and $E_{f}$ lead to the addition of nine modes in the fluid sub-basis. Note that the structure sub-basis does not require enrichment for the tolerance level under consideration. Equations (26) and (27) indicate that no enrichment is required by displacement residuals vectors. The basis $T^{E}$ is thus used to reduce the coupled model.

Table 1 Frequencies of coupled and uncoupled systems $(\mathrm{Hz})$

\begin{tabular}{lllll}
\hline$N^{\circ}$ & $\begin{array}{l}\text { Structure } \\
\text { Modes }\end{array}$ & $\begin{array}{l}\text { Acoustic } \\
\text { Modes }\end{array}$ & $\begin{array}{l}\text { Coupled } \\
\text { Modes }\end{array}$ & $\begin{array}{l}\text { Dominant } \\
\text { domain }\end{array}$ \\
\hline 1 & 147.50 & 0.00 & 0.00 & $\mathrm{f}$ \\
2 & 327.26 & 486.41 & 153.71 & $\mathrm{~s}$ \\
3 & 409.50 & 587.44 & 324.11 & $\mathrm{~s}$ \\
4 & 587.91 & & 406.34 & $\mathrm{~s}$ \\
5 & & & 489.53 & $\mathrm{f}$ \\
6 & & & 584.51 & $\mathrm{f}+\mathrm{s}$ \\
7 & & 590.12 & $\mathrm{f}+\mathrm{s}$ \\
\hline
\end{tabular}

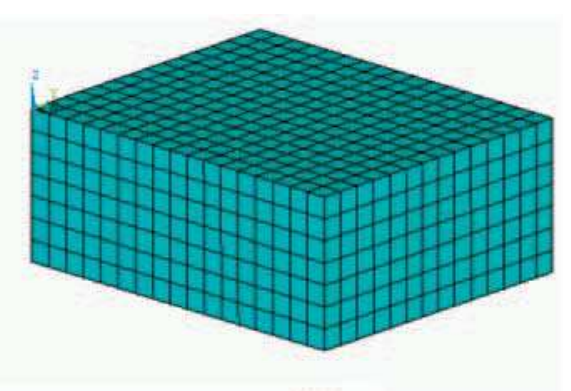

(b)

Fig. 2 Geometric model (a) and finite element model (b) 
The performance of the proposed method is evaluated relative to the reference method (direct method (DI)). The curves shown in Fig. 4 for the vibroacoustic indicators, respectively obtained by the proposed method and the direct method (reference), are in good agreement. The vibroacoustic indicators are predicted with errors about $0.002 \mathrm{~dB}$ for the sound power level and about $0.004 \mathrm{~dB}$ for the mean square velocity. In the coupling frequency band [585$590 \mathrm{~Hz}]$, which contains two coupled modes, the error reaches a maximum of $0.008 \mathrm{~dB}$.

Position of the proposed method with literature. As indicated in Section 4, the reduction basis should have the same size for all the methods. As a consequence, the computation time will be comparable. The maximum number of vectors in the structure sub-basis (Ns) and the fluid sub-basis (Nf) is used as a unified common size limit for all the methods. Figure 5 illustrates the vibroacoustic indicators. The uncoupled basis method (DB) and the uncoupled basis method with a priori selected modes (BDS) do not take into account the coupling effect. Thus, they have a relatively large error in the order of $10 \mathrm{~dB}$. The enrichment by the IR method is constrained by the common size limit of the sub-bases and would not reach its own stop criterion. It should be emphasized that, with only a few added residue vectors (which are eliminated here because of the size limit), this method would lead to very small errors compared with the reference case.
Table 2 indicates the computation time for each method. Referring to the computation time (CPU) for the DI method, the proposed method is more time-consuming than the $\mathrm{DB}$ and the BDS methods. The (IR) methods is the most timeconsuming.

The computational time compared to the prediction accuracy is a valuable criterion to estimate the efficiency of the method. As a result, for a weak coupling, the proposed method predicts the vibroacoustic indicators with an error of about $0.004 \mathrm{~dB}$ and a reduction in computation time by 89 per cent over a complete model.

\subsubsection{Case of cavity filled with water}

The interest of the proposed method is greater in the case of strong coupling. The example of the rectangular cavity is considered again with the same dimensions. To simulate a strong coupling, the cavity is filled with water.

Table 3 shows the first four structure eigenfrequencies and only one fluid eigenfrequency in the frequency band of interest. The eigenfrequencies of the coupled system are due to the coupling of the higher rank fluid modes and structural modes. In the same band, the first nine eigenfrequencies of the coupled fluid-structure system are presented. We note that, in the strong coupling case, many modes exhibit coupled fluid-structure behaviour.
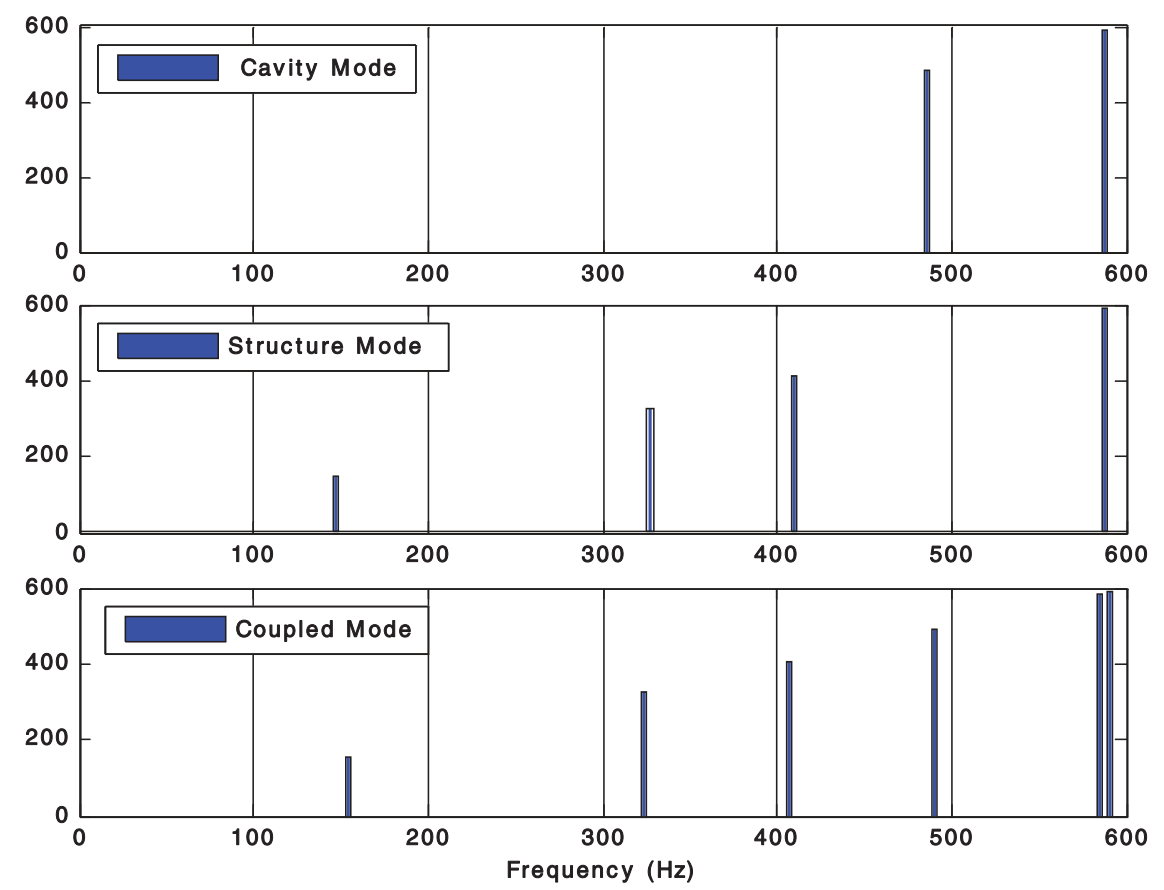

Fig. 3 Eigenfrequencies of coupled and uncoupled systems 

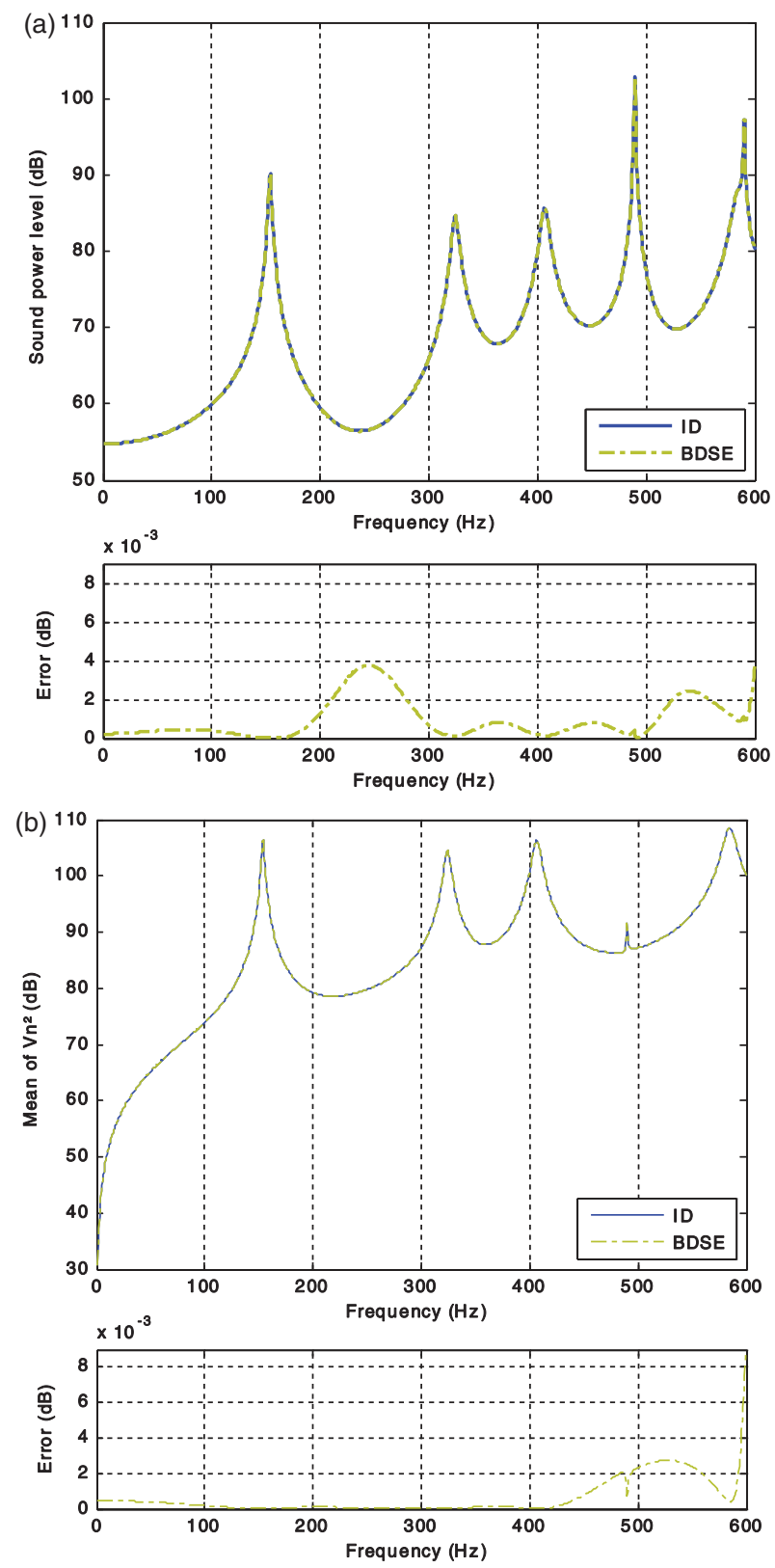

Fig. 4 Performance of the proposed method with weak coupling

Validation of the proposed method. As presented in the weak coupling cavity example, equations (4) are exploited to build the uncoupled fluid-structure sub-bases. To start with, the calculation of 200 structure modes and 200 fluid modes is carried out. Next, indicator (8) is used to rank the useful modes among the retained ones. Following this, 25 structural modes and 108 fluid modes are selected by using the criteria $E_{s}$ and $E_{f}$ (equations (19) and (20)) with a tolerance $\mathrm{Tol}=10^{-3}$. The truncation criteria for the structure (a) and fluid (b) sub-bases are shown in the Fig. 6.

Subsequently, the use of equations (23) associated with criteria $E_{s}$ and $E_{f}$, estimated with a
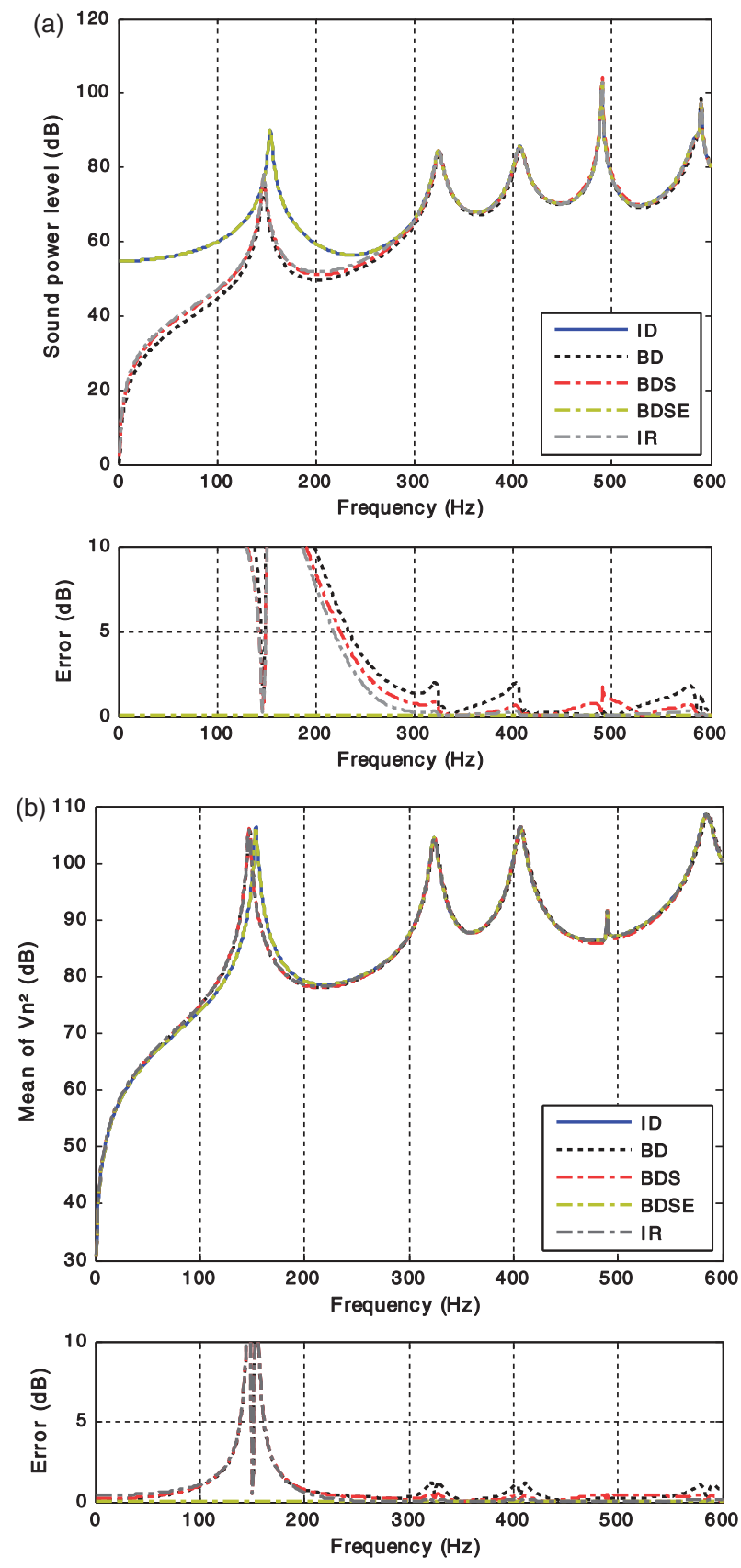

Fig. 5 Vibroacoustic indicators (weak coupling)

tolerance $\mathrm{Tol}=10^{-3}$, improves the structure subbasis by adding 21 vectors while the fluid subbasis has 39 new vectors. Figure 7 shows the structure (a) and fluid (b) enrichment indicators; it illustrates that the indicators are established at the chosen tolerance.

Finally, equations (26) and (27) associated with the criteria $E_{s}$ and $E_{f}$ act as stopping criteria to quantify the accuracy of the final basis. This test will serve as a decision tool for adding one structural and one fluid displacement residual vectors. The final enriched basis $T^{E}$ is used for the coupled model reduction. Unlike the case of weak coupling, both structure 
and fluid sub-bases must be enriched for the strong coupling.

Figure 8 shows a superposition of the coupled vibroacoustic indicators, relative to the direct

Table 2 CPU Time (seconds) for a weak coupling

\begin{tabular}{llllll}
\hline & ID & BD & BDS & IR & BDSE \\
\hline CPU (s) & 1639 & 28 & 1100 & 2100 & 1200 \\
Ratio (\%) & 100 & 1 & 50 & 95 & 55 \\
\hline
\end{tabular}

Table 3 Frequencies of coupled and uncoupled systems

\begin{tabular}{lllll}
\hline$N^{\circ}$ & $\begin{array}{l}\text { Structure } \\
\text { Modes }\end{array}$ & $\begin{array}{l}\text { Acoustic } \\
\text { Modes }\end{array}$ & $\begin{array}{l}\text { Coupled } \\
\text { Modes }\end{array}$ & $\begin{array}{l}\text { Dominant } \\
\text { domain }\end{array}$ \\
\hline 1 & 147.50 & 0.00 & 0.00 & $\mathrm{f}$ \\
2 & 327.26 & - & 94.40 & $\mathrm{~s}+\mathrm{f}$ \\
3 & 409.50 & - & 131.05 & $\mathrm{~s}+\mathrm{f}$ \\
4 & 587.91 & - & 220.26 & $\mathrm{~s}+\mathrm{f}$ \\
5 & - & - & 237.43 & $\mathrm{~s}+\mathrm{f}$ \\
6 & - & - & 351.72 & $\mathrm{~s}+\mathrm{f}$ \\
7 & - & - & 384.45 & $\mathrm{~s}+\mathrm{f}$ \\
8 & - & - & 452.94 & $\mathrm{~s}+\mathrm{f}$ \\
9 & - & - & 484.57 & $\mathrm{~s}+\mathrm{f}$ \\
\hline
\end{tabular}
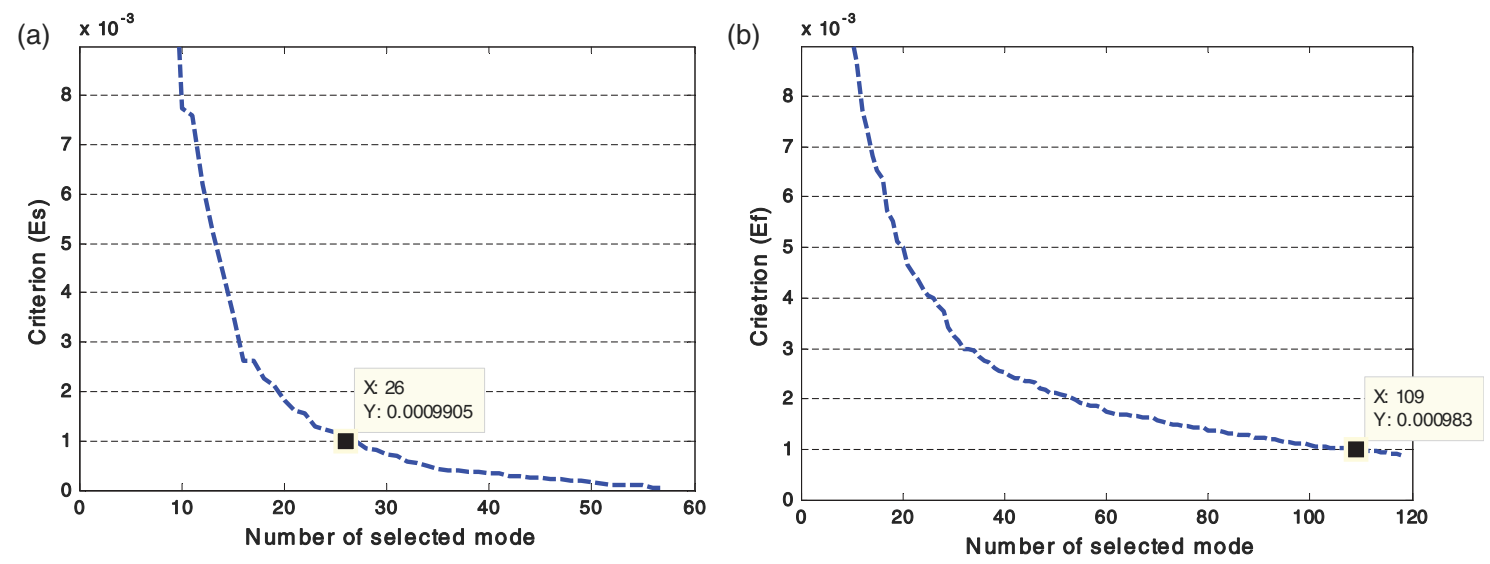

Fig. 6 Structure (a) and fluid (b) sub-bases truncation criteria
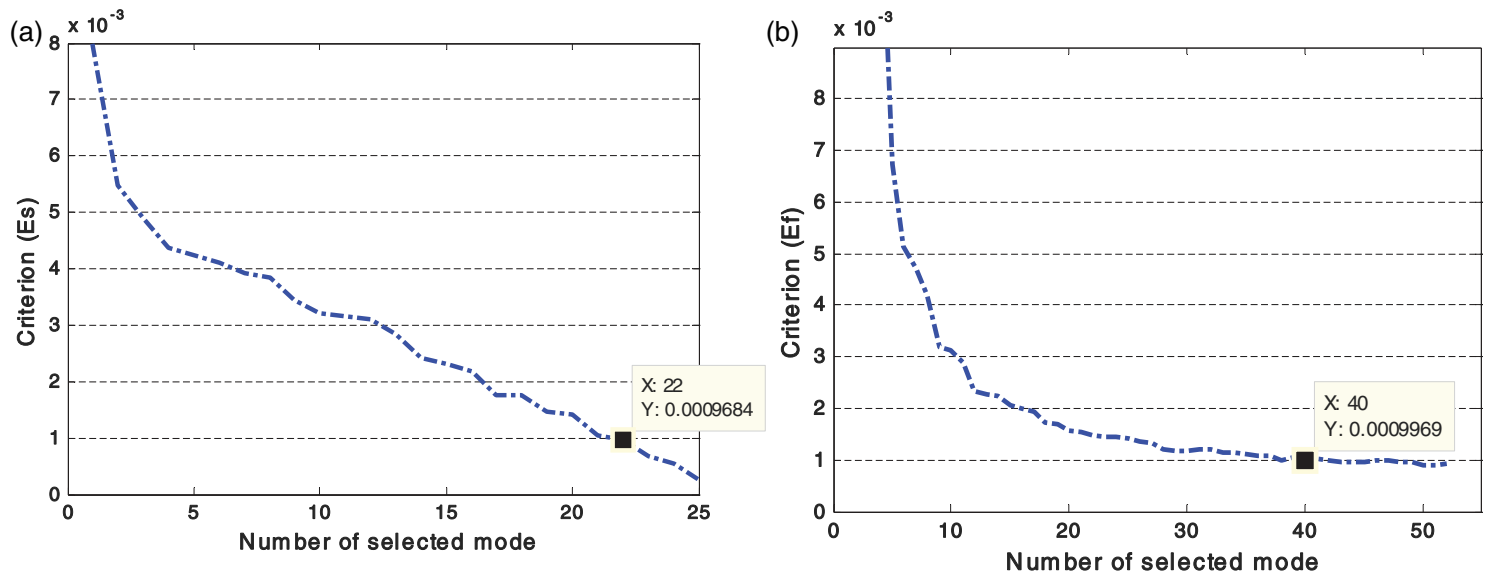

Fig. 7 Structure (a) and fluid (b) sub-bases enrichment criteria 

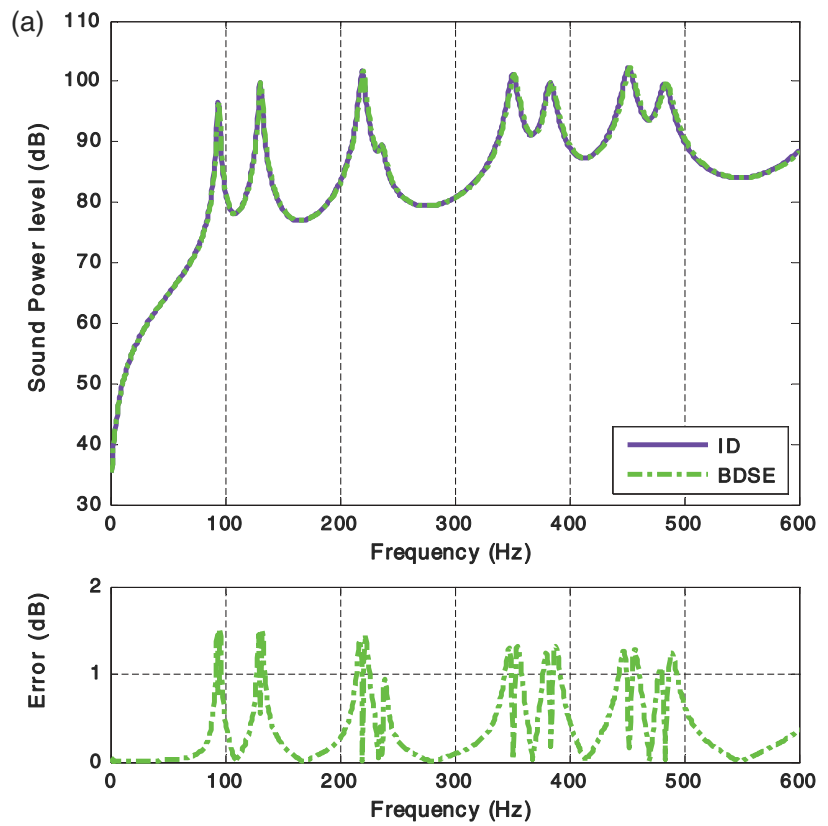

(b)
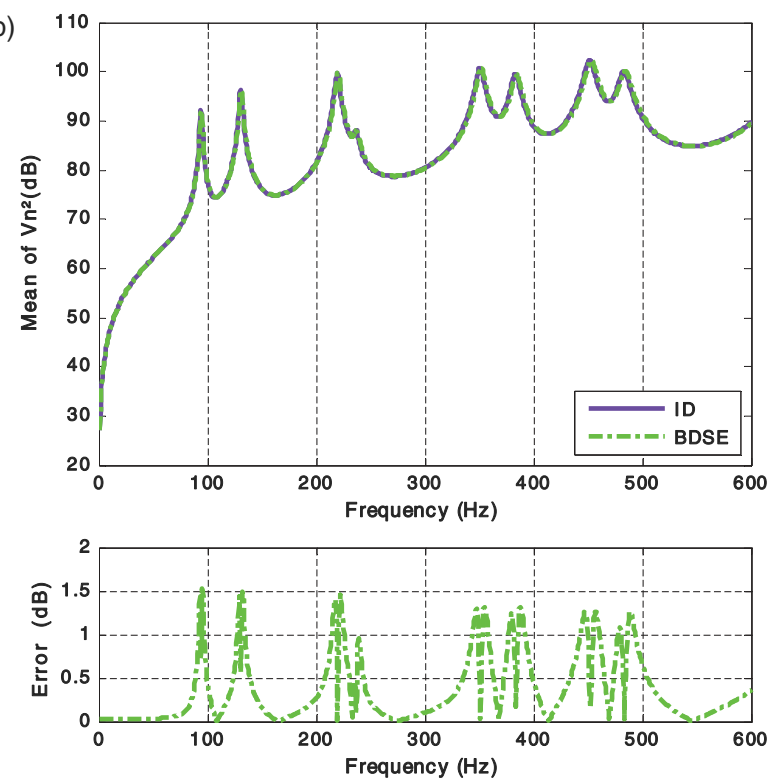

Fig. 8 Performance of the proposed method with strong coupling

curves with various accuracies and tolerances. Tables 4 and 5 shows the computation cost, the model reduction size, the mean error and the eigenfrequency for each method. We note that the computation cost increases with accuracy.

Position of the proposed method within the literature. The same tolerance is used for all the methods Tol $=10^{-3}$. The vibroacoustic indicators are shown in Fig. 10. The methods BD and BDS, as in the weak coupling case, are not able to reconstruct vibroacoustic indicators obtained by the reference method (ID). For the cavity filled with water, they do not take into account the strong coupling effects.
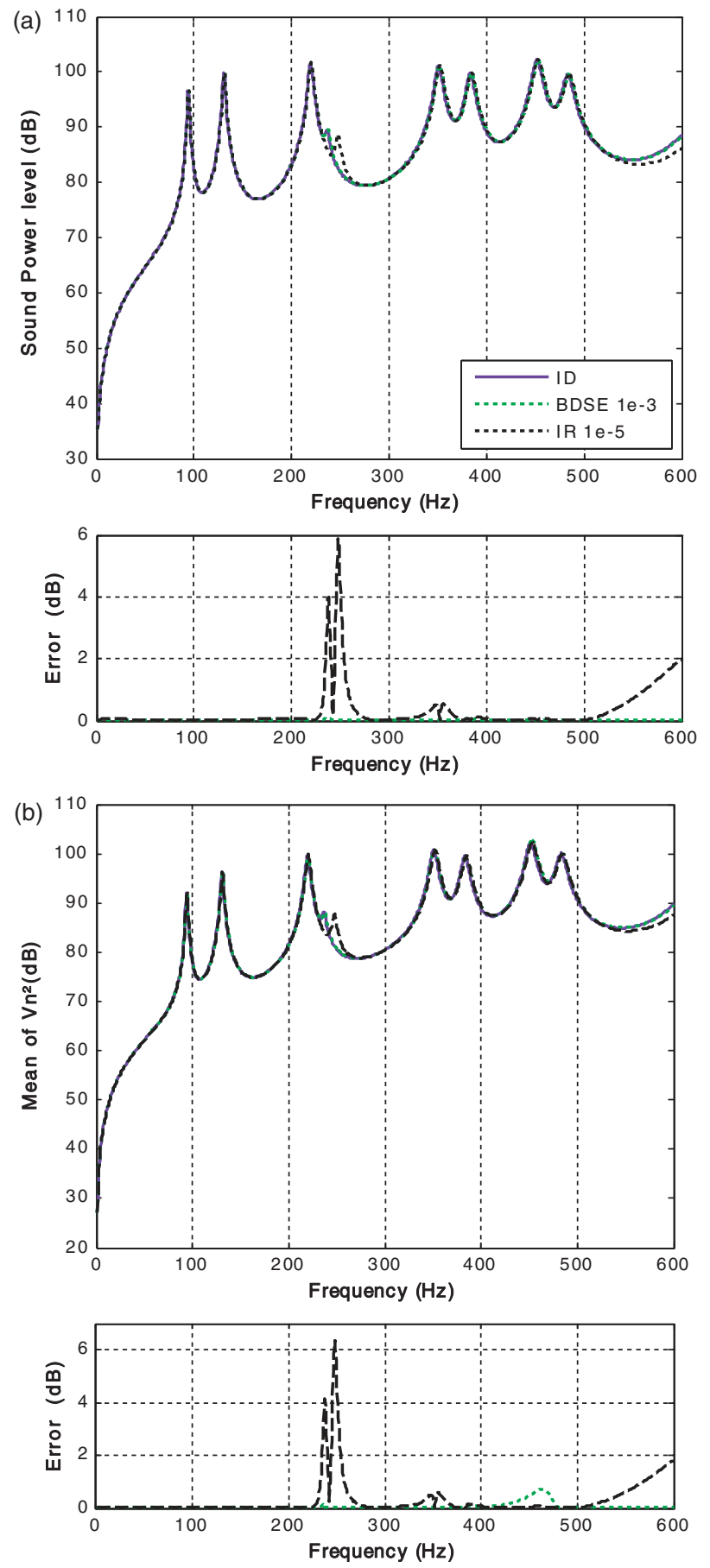

Fig. 9 Vibro-acoustic indicators using (IR: 224 DOFs), (BDSE: 209 DOFs), and (ID: 3324 DOFs) methods

The proposed BDSE and the IR methods need more time to obtain the enriched bases. But in this case the last one is once again constrained by the sub-basis size limit.

Table 6 indicates the computation time for each method. referring to the computation time (CPU) for the (DI) method. The ratio of time-consumption is 59 per cent for the proposed method and 95 per 
Table 4 IR and BDSE methods performance

\begin{tabular}{llll}
\hline & $\begin{array}{l}\text { Reduced Model } \\
\text { Size (DOFs) }\end{array}$ & $\begin{array}{l}\text { Mean } \\
\text { error (db) }\end{array}$ & $\begin{array}{l}\text { CPU } \\
(\mathrm{s})\end{array}$ \\
\hline IR Method & 224 & 1 & 4087 \\
BDSE Method & 209 & 0.2 & 1258 \\
Ratio=BDSE/IR(\%) & 93 & 20 & 31 \\
\hline
\end{tabular}

Table 5 IR and BDSE methods performance for eigenfrequency computing

\begin{tabular}{|c|c|c|c|c|c|}
\hline \multirow[b]{2}{*}{$\mathrm{N}^{\circ}$} & \multirow{2}{*}{$\begin{array}{l}\text { Complete } \\
\text { Model } \\
\text { (3324 DOFs) }\end{array}$} & \multicolumn{2}{|c|}{$\begin{array}{l}\text { Reduced Model IR } \\
\left(224 \text { DOFs, } T o l=10^{-3}\right.\end{array}$} & \multicolumn{2}{|c|}{$\begin{array}{l}\text { Reduced Model BDSE } \\
\text { (209DOFs, } T o l=10^{-3}\end{array}$} \\
\hline & & $f(H z)$ & $\epsilon_{v}(\%)$ & $f(H z)$ & $\epsilon_{v}(\%)$ \\
\hline 1 & 0.00 & 0.00 & 0.00 & 0.00 & 0.00 \\
\hline 2 & 94.40 & 94.40 & 0.00 & 94.40 & 0.00 \\
\hline 3 & 131.05 & 131.05 & 0.00 & 131.05 & 0.00 \\
\hline 4 & 220.26 & 220.27 & 0.00 & 220.26 & 0.00 \\
\hline 5 & 237.43 & 247.56 & 0.04 & 237.52 & 0.00 \\
\hline 6 & 351.72 & 352.21 & 0.00 & 351.73 & 0.00 \\
\hline 7 & 384.45 & 384.56 & 0.00 & 384.50 & 0.00 \\
\hline 8 & 452.94 & 453.03 & 0.00 & 452.93 & 0.00 \\
\hline 9 & 484.57 & 484.62 & 0.00 & 484.56 & 0.00 \\
\hline
\end{tabular}

Table 6 CPU Time (seconds) for a strong coupling

\begin{tabular}{llllll}
\hline & ID & BD & BDS & IR & BDSE \\
\hline CPU (s) & 2200 & 28 & 1100 & 2100 & 1300 \\
Ratio (\%) & 100 & 1 & 50 & 95 & 59 \\
\hline
\end{tabular}

cent for the IR methods. They are usually more timeconsuming compared to the weak coupling case, because several enrichment vectors are added to the reduction basis.

The proposed method reduces the computation time by 40 per cent with an accuracy that does not exceed $1.5 \mathrm{~dB}$ compared with the complete model. The error level could be reduced by introducing a lower tolerance threshold for the initial modes selection and for the enrichment step.

\subsection{Strong coupling case with pipe geometry}

The second example is devoted to the validation of the proposed method for more complex geometric shapes and larger model size. The system is composed of two tubes, $0.003 \mathrm{~m}$ in thickness. The small tube is $R_{1}=0.1 \mathrm{~m}$ in radius and $\mathrm{L}_{1}=0.4 \mathrm{~m}$ in length. The tube with larger diameter is $R_{2}=0.25 \mathrm{~m}$ in radius and $\mathrm{L}_{2}=1 \mathrm{~m}$ in length. An intermediate plate provides the connection of the two cylinders. The pipe ends are rigid walls, and the structure is simply supported. The structure and fluid properties are the same as those used in the cavity example for the case of strong coupling. To ensure maximum
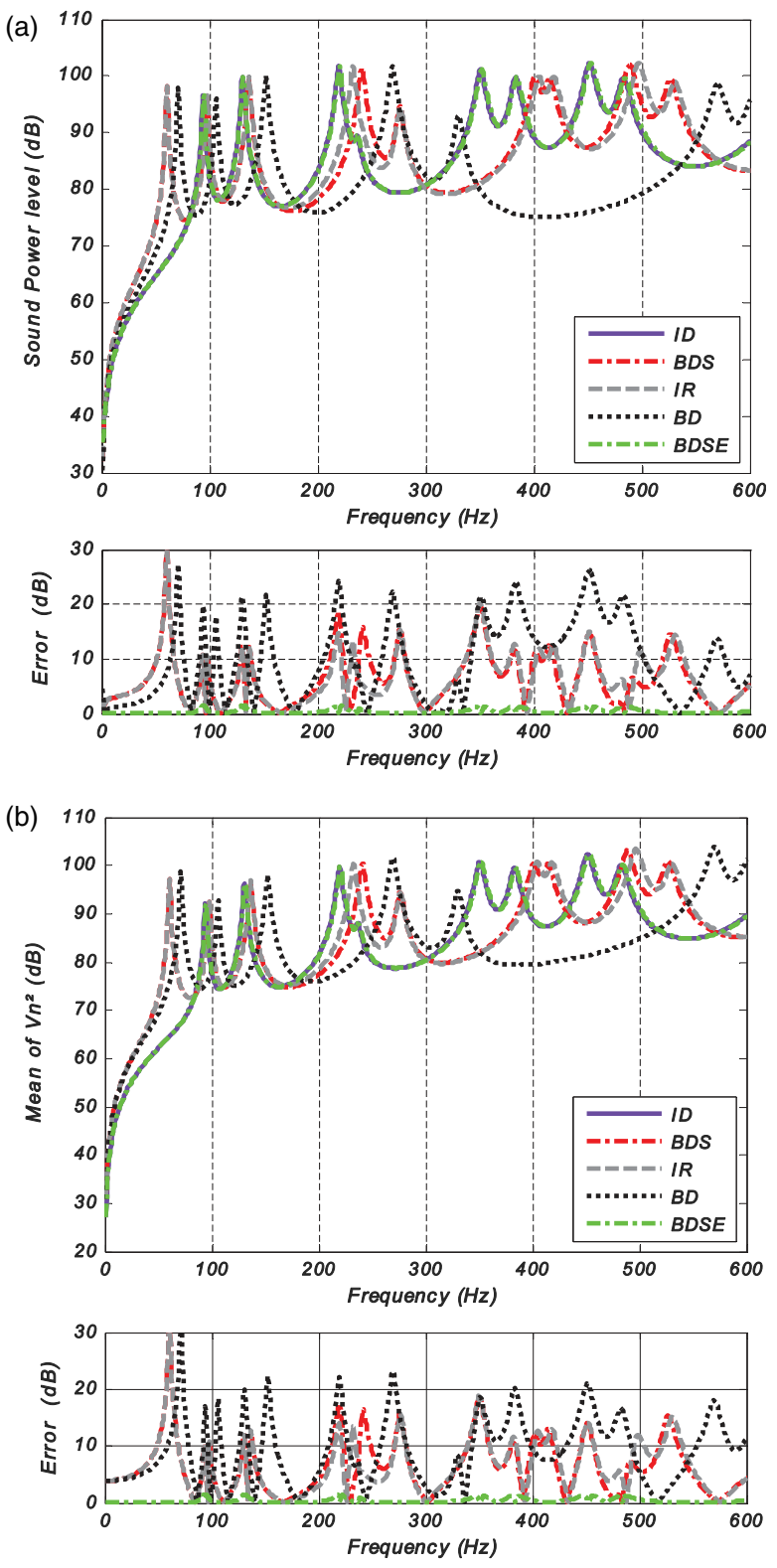

Fig. 10 Vibroacoustic indicators (case of strong coupling)

excitability, two point harmonic forces are applied as shown in Fig. 11(b). The full finite element model has 6544 DOFs (3024 structural DOFs and 3520 fluid DOFs). The mesh of the finite elements model is based on a criterion of five to six linear elements per mode shape wavelength. This criterion is used by Boily et al. [13].

The frequency range of interest in this example is [50-200Hz] containing the first eight modes of the coupled system illustrated in Table 7 . These modes are the results of the coupling effects with modes of higher rank outside the useful band.

One can proceed as in the parallelepipedic cavity example to describe the steps of the proposed 
(a)

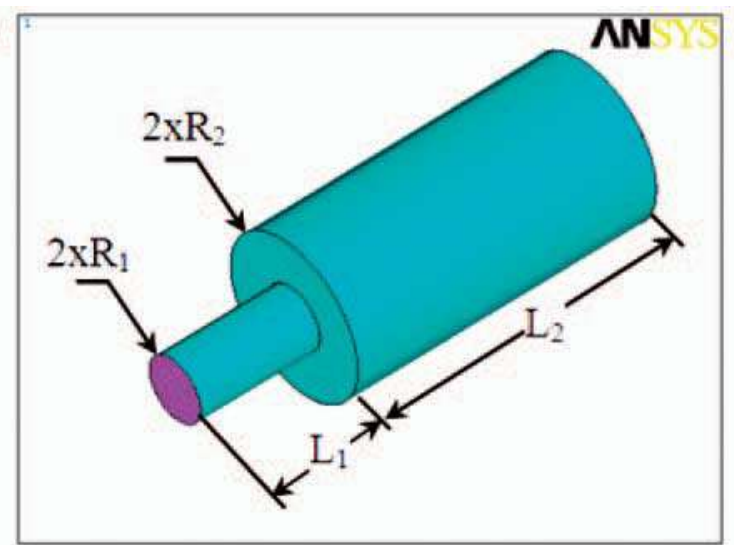

(b)

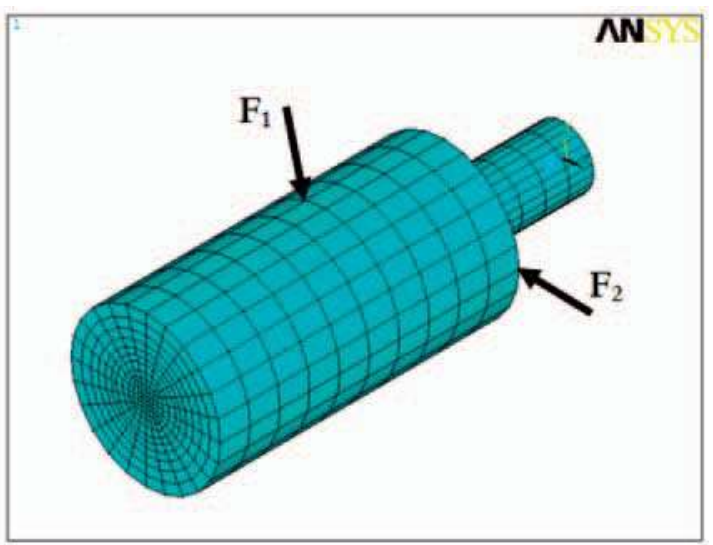

Fig. 11 Geometric (a) and finite element (b) model for the pipe with strong coupling

Table 7 Frequencies of coupled and uncoupled systems

\begin{tabular}{lllll}
\hline$N^{\circ}$ & $\begin{array}{l}\text { Structure } \\
\text { Modes }\end{array}$ & $\begin{array}{l}\text { Acoustic } \\
\text { Modes }\end{array}$ & $\begin{array}{l}\text { Coupled } \\
\text { Modes }\end{array}$ & $\begin{array}{l}\text { Dominant } \\
\text { domain }\end{array}$ \\
\hline 1 & - & 0.00 & 0.00 & $\mathrm{f}$ \\
2 & - & - & 95.79 (double) & $\mathrm{s}+\mathrm{f}$ \\
3 & - & - & 97.13 (double) & $\mathrm{s}+\mathrm{f}$ \\
4 & - & - & 109.62 (double) & $\mathrm{s}+\mathrm{f}$ \\
5 & - & - & 134.62 (double) & $\mathrm{s}+\mathrm{f}$ \\
6 & - & - & 136.06 (double) & $\mathrm{s}+\mathrm{f}$ \\
7 & - & - & 165.35 & $\mathrm{~s}+\mathrm{f}$ \\
8 & - & - & 180.32 (double) & $\mathrm{s}+\mathrm{f}$ \\
\hline
\end{tabular}

method. One can start with the construction of the uncoupled structure sub-basis $T_{\text {so }}$ and fluid sub-basis $T_{f o}$ truncated at the size of 200 structure and fluid modes. Next, the retained modes are ranked with the indicator (8). Afterward, 48 structure modes and 143 fluid modes are selected corresponding to a tolerance $\mathrm{Tol}=10^{-3}$. After that, the structure basis is extended by 36 modes and the fluid basis is extended by 45 modes.

In the final step, one structure and one fluid displacement residuals vector are added to achieve an accuracy of the final basis of $10^{-8}$. The final enriched basis $T^{E}$ is used to reduce the coupled complete model.

Figure 10 shows a superposition of the coupled vibroacoustic indicators obtained by using the uncoupled basis $T^{0}$ (BD) and enriched $T^{E}$ (BDSE) and their errors compared with the reference method based on direct inversion of dynamic stiffness (DI). This example illustrates the interest of the proposed method for structures with relatively complex geometry and large finite element model size. The method can predict the vibroacoustic indicators obtained with errors around $0.5 \mathrm{~dB}$ for the mean square velocity and $1 \mathrm{~dB}$ for the acoustic power (Fig. 12). In Table 8, there is a significant reduction
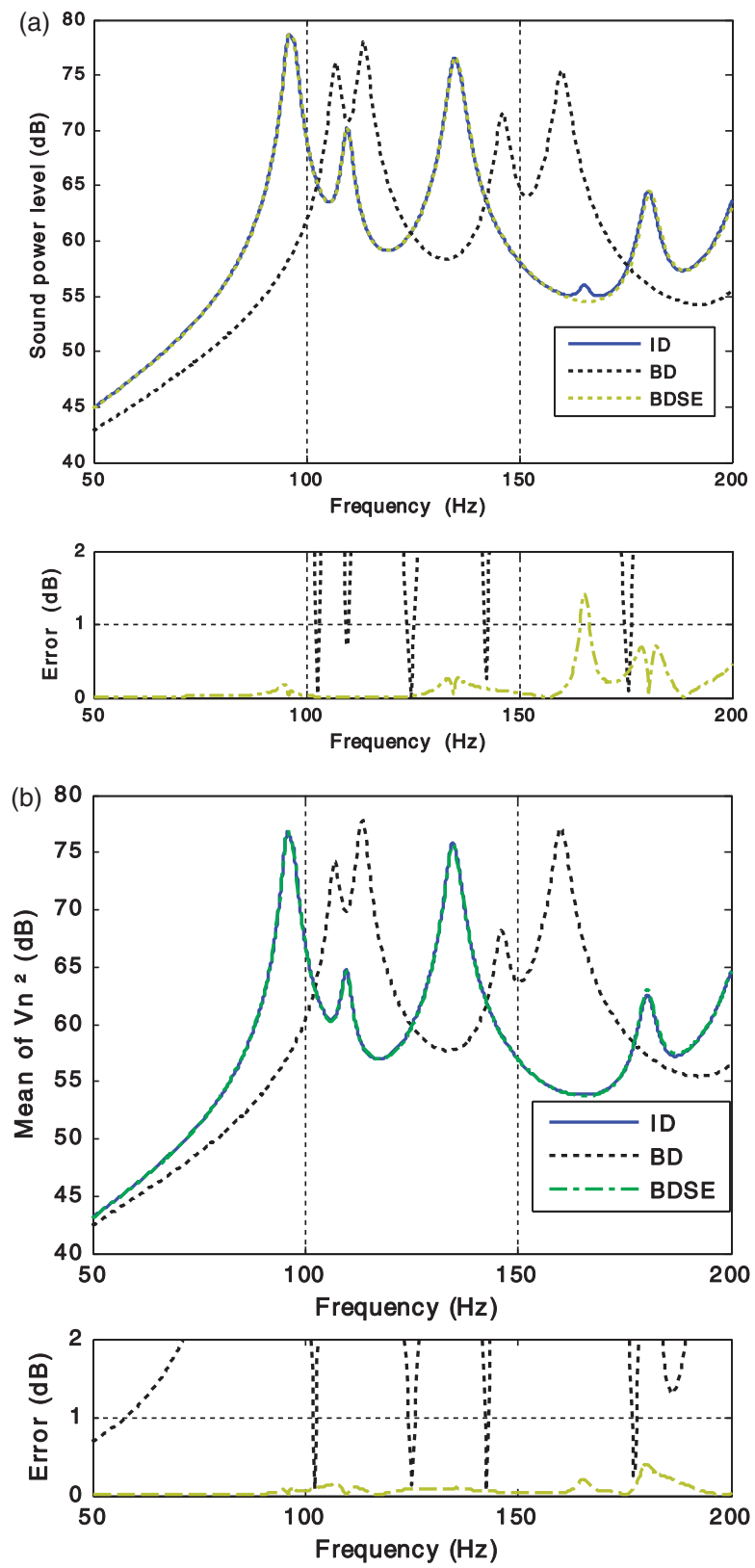

Fig. 12 Vibroacoustic indicators (strong coupling) 
Table 8 CPU time (seconds) for a pipe strong coupling

\begin{tabular}{llll}
\hline & ID & BD & BDSE \\
\hline CPU (s) & 5835 & 25 & 2400 \\
Ratio (\%) & 100 & 1 & 41 \\
\hline
\end{tabular}

in computational time compared with the direct inversion method (ID). For the proposed method the reduction ratio is about 40 per cent.

\section{CONCLUSION}

In this paper, a reduction method for coupled vibroacoustic problems is proposed. This method is based on a priori selection of useful modes in the fluid and structure truncated sub-bases. These modes contribute significantly in the vibroacoustic indicators prediction. The sub-bases are enriched by terms taking into account the coupling and the fluid-structure interaction. The numerical simulations show that the accuracy of the reduced model, for both weak and strong coupled fluidstructure systems, are significantly improved. The method exhibits interesting performance in terms of computation time (CPU) and overall accuracy of vibroacoustic indicators. The work in progress associated to the proposed method is related to non-linear coupled fluid-structure analysis, optimization techniques and uncertainties propagation in robustness analyses, which are all known to require high CPU time.

(C) Authors 2011

\section{REFERENCES}

1 Pretlove, A. J. Forced vibrations of a rectangular panel backed by a closed rectangular cavity. J. Sound Vibrat., 1966, 3, 252-261.

2 Fischer, M. The fast multipole boundary element method and its application to structure-acoustic field interaction, 2004 (Bericht Mechanical Institute, Stuttgart University).

3 Lesueur, C. Rayonnement acoustique des structures, vibroacoustique, interactions fluide-structures, 1988 (Paris, Eyrolles).

4 Morand, H. J. P. and Ohayon, R. Fluid structure interaction, 1995 (Edition Masson).

5 Ohayon, R. and Soize, C. Structural acoustics and vibration, 1998 (Academic Press).

6 Ohayon, R. Linear vibrations of structures coupled with an internal fluid. In Eccomas School 2006 (Ibiza, Spain).

7 Irons, B. M. Role of part-inversion in fluid-structure problems with mixed variables. AIAA J., 1970, 8(3), 568.
8 Felippa, C. A. Symmetrization of the contained compressible-fluid vibration eigenproblem. Comm. Appl. Num. M., 1985, 1, 241-247.

9 Tran, Q. H., Ouisse, M., and Bouhaddi, N. A robust component mode synthesis method for stochastic damped vibro-acoustics. Mech. Syst. Signal Pr., 2010, 24, 164-181.

10 Dhandolen, S. D. and Modak, S. V.Review of vibroacoustics analysis procedures for prediction of low frequency noise inside a cavity, International Modal Analysis Conference (IMAC), Orlando, Florida, USA, 2007.

11 Bobillot, A. Méthode de réduction pour le recalage, Application au cas d'Ariane 5. thèse de doctorat en mécanique, Ecole Centrale de Paris 2002.

12 Davidsson, P. Structure-acoustic analysis, Finite element modeling and reduction methods, Doctoral thesis, Structural Mechanics, Lund University, 2004.

13 Boily, S. and Charron, F. The vibroacoustic response of a cylindrical shell structure with viscoelastic and poroelastic materials. Appl. Acoust., 1999, 58, 131-152.

14 Boily, S. Etude numérique du comportement vibroacoustique de coques semi-complexe pour une excitation solidienne, 1996 (maitrize en génie mécanique, Université, Sherbrooke).

15 Tournour, M. and Atalla, N. Pseudostatic corrections for the forced vibroacoustic response of a structurecavity system. J. Acoust. Soc., 2000, 107, 2379-2386.

16 Maess, M. and Gaul, L. Substructing and model reduction of pipe components interacting with acoustic fluids. Mech. Syst. Signal Pr., 2006, 20, 45-64.

17 Géradin, M. and Rixen, D. Mechanical vibrations: Theory and applications to structural dynamics, 1997 (Wiley, New York).

\section{APPENDIX}

\section{Notation}

$U$ displacement vector, physical coordinates

$P \quad$ pressure vector, physical coordinates

$M \quad$ mass matrix

$K \quad$ stiffness matrix

$C \quad$ coupling matrix

$F \quad$ external force vector

$\rho_{f} \quad$ fluid density

$T_{s} \quad$ structure domain reduction basis

$T_{f} \quad$ fluid domain reduction basis

$q_{s} \quad$ displacement vector, generalized coordinates

$q_{f} \quad$ pressure vector, generalized coordinates

$E_{s} \quad$ structure basis criterion

$R \quad$ residual vector

$E_{f} \quad$ fluid basis criterion

$\omega \quad$ angular frequency ( $\mathrm{rad} / \mathrm{s})$

$f \quad$ frequency $(\mathrm{Hz})$

(.) item for reduced matrix and vectors

(.) item for damped matrix

(.) item for shifted matrix 